\title{
Efficacy of sentinel lymph node biopsy with radioisotope alone and the prediction of sentinel node status using PET-CT in breast cancer
}

\author{
Ran Song, Seong Uk Kwon, Dae Sung Yoon, In Eui Bae, In Seok Choi, Won Jun Choi, Sang Eok Lee, Ju Ik Moon, Nak Song Sung, \\ Seung Jae Lee, Seung Jae Roh, Sung Gon Kim \\ Department of Surgery, Konyang University Hospital, Daejeon, Korea
}

Purpose: Sentinel lymph node biopsy (SLNB) using both a radioactive isotope (RI) and blue dye is considered highly effective; however, there were limitations with the use of both agents in some hospitals, and blue dye has been shown to have some adverse effects. Additionally, preoperative prediction of sentinel lymph node (SLN) status using the maximum standardized uptake value (SUVmax) on positron emission tomography-computed tomography (PET-CT) can help avoid unnecessary axillary dissection or SLNB. Thus, we evaluated the efficacy and oncologic safety of SLNB using an RI alone in terms of long-term outcomes and determined the association between SLN metastasis and SUVmax of the primary tumor.

Methods: This retrospective study was conducted at Konyang University Hospital between March 2011 and May 2018. Overall, 142 patients with breast cancer who underwent SLNB using an RI alone were enrolled. Data on identification and false-negative rates were collected. The SUVmax of primary tumors on PET-CT were analyzed for their association with SLN metastasis.

Results: The identification and false-negative rates were $98.6 \%$ and $0 \%$, respectively. There was no axillary local recurrence in patients with negative SLN findings. The correlation between the SUVmax of the primary tumor and SLN status was significant $(r=0.249, P=0.005)$; the cutoff value for negative SLN metastasis was $<2.15$.

Conclusion: The single agent method using an RI is not inferior to other methods and serves as a feasible option for SLNB. And the number of excised SLNs could be minimized when the SUVmax of primary tumor is extremely low.

Keywords: Breast neoplasms, Sentinel lymph node biopsy, Radioisotope, Positron emission tomography computed tomography

Received: Apr 9, 2021 Revised: Jun 24, 2021 Accepted: Jun 26, 2021 Correspondence to: Seong Uk Kwon

Department of Surgery, Konyang University Hospital, 158 Gwanjeodongro, Seo-gu, Daejeon 35365, Korea

Tel: +82-42-600-8956, Fax: +82-42-543-8956

E-mail: ksu2017@kyuh.ac.kr

ORCID: Ran Song (https://orcid.org/0000-0001-6373-7411), Seong Uk Kwon (https://orcid.org/0000-0003-3167-7527), Dae Sung Yoon (https://orcid.org/00000002-6447-2862), In Eui Bae (https://orcid.org/0000-0002-9220-8815), In Seok Choi (https://orcid.org/0000-0002-9656-3697), Won Jun Choi (https://orcid. org/0000-0003-0788-7700), Sang Eok Lee (https://orcid.org/0000-0003-27668791), Ju Ik Moon (https://orcid.org/0000-0002-8120-5854), Nak Song Sung (https://orcid.org/0000-0002-7549-3829), Seung Jae Lee (https://orcid.org/00000002-3302-6624), Seung Jae Roh (https://orcid.org/0000-0002-3232-2798), Sung Gon Kim (https://orcid.org/0000-0002-8837-073X)

Copyright @ 2021 Korean Society of Surgical Oncology

This is an Open Access article distributed under the terms of the Creative Commons Attribution Non-Commercial License (http://creativecommons.org/licenses/by-nc/4.0) which permits unrestricted non-commercial use, distribution, and reproduction in any medium, provided the original work is properly cited.

\section{INTRODUCTION}

Breast cancer is the most common cancer and the main cause of cancer-related deaths in women worldwide [1,2]. Sentinel lymph node biopsy (SLNB) is a standard method for axillary assessment in breast cancer patients without clinical axillary metastases $[3,4]$. SLNB can be used to avoid unnecessary axillary lymph node dissection (ALND), thereby minimizing morbidities such as pain, lymphedema, and limitation of arm motion $[5,6]$. Radioactive isotopes (RIs), blue dye, or both are generally used for sentinel lymph node (SLN) assessment $[3,7,8]$. Many studies have compared the efficacy of the agents for SLN assessment [9-13]. In general, the combined method involving an RI and blue dye is more effective for identifying SLNs than an RI or blue dye alone $[9,12,13]$. Therefore, most high-volume centers in Seoul, South Korea, currently use both an RI and blue dye in SLNB. However, there are some limitations to using the combined method. First, allergic or ana- 
phylactic reactions have been reported during SLNB using blue dye in previous studies $[11,14]$. Temporary blue staining [15] and capsular contraction [16] were also found in patients who underwent SLNB using blue dye. Additionally, some hospitals lack the equipment or facilities needed to use the combined method. Furthermore, some previous studies have shown that there was no statistical difference in the false-negative rate between the combined method and the method using an RI alone $[9,10,12]$. For these reasons, some hospitals, including our institution, use an RI alone in SLNB. However, it is unclear how effective or safe this method is. Therefore, it is necessary to evaluate the efficacy and oncologic safety of SLN assessment using an RI alone.

Additionally, some studies have suggested an association between the maximum standardized uptake value (SUVmax) of the tumor on positron emission tomography-computed tomography (PET-CT) and axillary lymph node (ALN) metastasis in breast cancer patients [17-19]. These studies have suggested that a higher SUVmax of the primary tumor or a higher ALN-to-tumor ratio was associated with a greater risk of ALN metastasis [17-19]. Preoperative prediction of SLN status can help avoid unnecessary procedures such as ALND and even SLNB.

Thus, this study aimed to evaluate the efficacy and oncologic safety of SLNB using RI alone with respect to long-term clinical outcomes in breast cancer patients and to determine the association between SLN status and the SUVmax of primary tumors on PET-CT.

\section{METHODS}

\section{Patient selection}

Data were retrospectively collected at Konyang University Hospital between March 2011 and May 2018. A total of 184 patients who underwent SLNB with an RI alone were enrolled in this study. All patients were histopathologically diagnosed with invasive breast cancer with negative ALNs based on physical examinations and imaging studies such as ultrasonography and magnetic resonance imaging. Eighteen patients with $<24$ months of follow-up and 24 patients who underwent ALND without SLNB because of failure of SLN identification or the presence of ALNs with suspected metastasis in the operating field were excluded from this study. However, two patients with failure of SLN identification were included only in the calculation of the identification rate. Patients with 10 or more harvested lymph nodes were considered to have undergone ALND and therefore, were excluded. Because there were no clear criteria for the upper limit of the number of SLNs, we classified patients with 10 or more harvested lymph nodes as those who underwent ALND according to a few previous studies that suggested

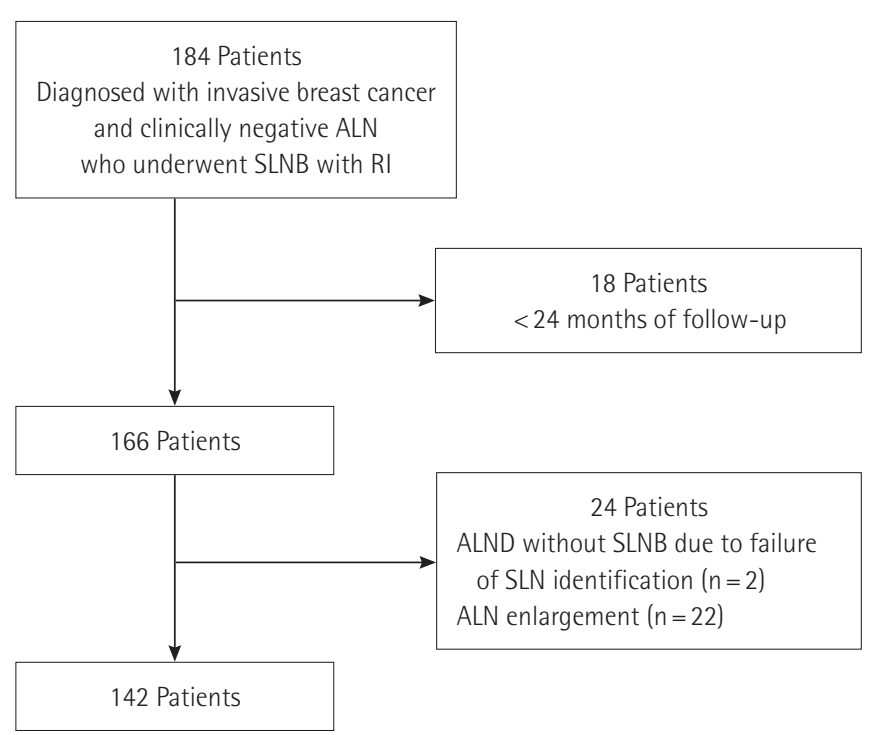

Fig. 1. Flowchart showing patient selection. ALN, axillary lymph node; SLNB, sentinel lymph node biopsy; $\mathrm{Rl}$, radioactive isotope; ALND, axillary lymph node dissection; SLN, sentinel lymph node.

that at least 10 lymph nodes were required to be harvested for proper ALND [20,21]. Finally, data of 142 patients who met the inclusion criteria were analyzed (Fig. 1). The study was approved by the Institutional Review Board of Konyang University Hospital (IRB No. 2019-07-015) and performed in accordance with the principles of the Declaration of Helsinki. The need for informed consent was waived.

\section{Identification of SLNs}

In all cases, SLNs were assessed using RI guidance. We injected 0.2 $\mathrm{mL}$ of ${ }^{99 \mathrm{~m}} \mathrm{Tc}$-phytate at four sites of subcutaneous tissue around the areola preoperatively. After injection, gentle breast massage for 5 minutes was performed to facilitate migration of the particles to the axilla. Preoperative lymphoscintigraphy was performed to identify the drainage of SLNs (Fig. 2). SLNs were intraoperatively detected using a hand-held gamma probe and examined using frozen biopsy. ALND was performed in patients showing SLN metastasis on frozen biopsy or firm and enlarged ALNs that may suspect metastasis in the operating field.

\section{Data collection}

Demographic data, including data on age, sex, and menopausal state, and clinicopathologic data, such as data on tumor size, laterality, histologic type, histologic grade, hormone receptors (estrogen receptor $[\mathrm{ER}]$ /progesterone receptor $[\mathrm{PR}]$ ), and human epidermal growth factor receptor 2 (HER2) status, were collected. Data for evaluating the efficacy of SLNB, including data on the identifica- 


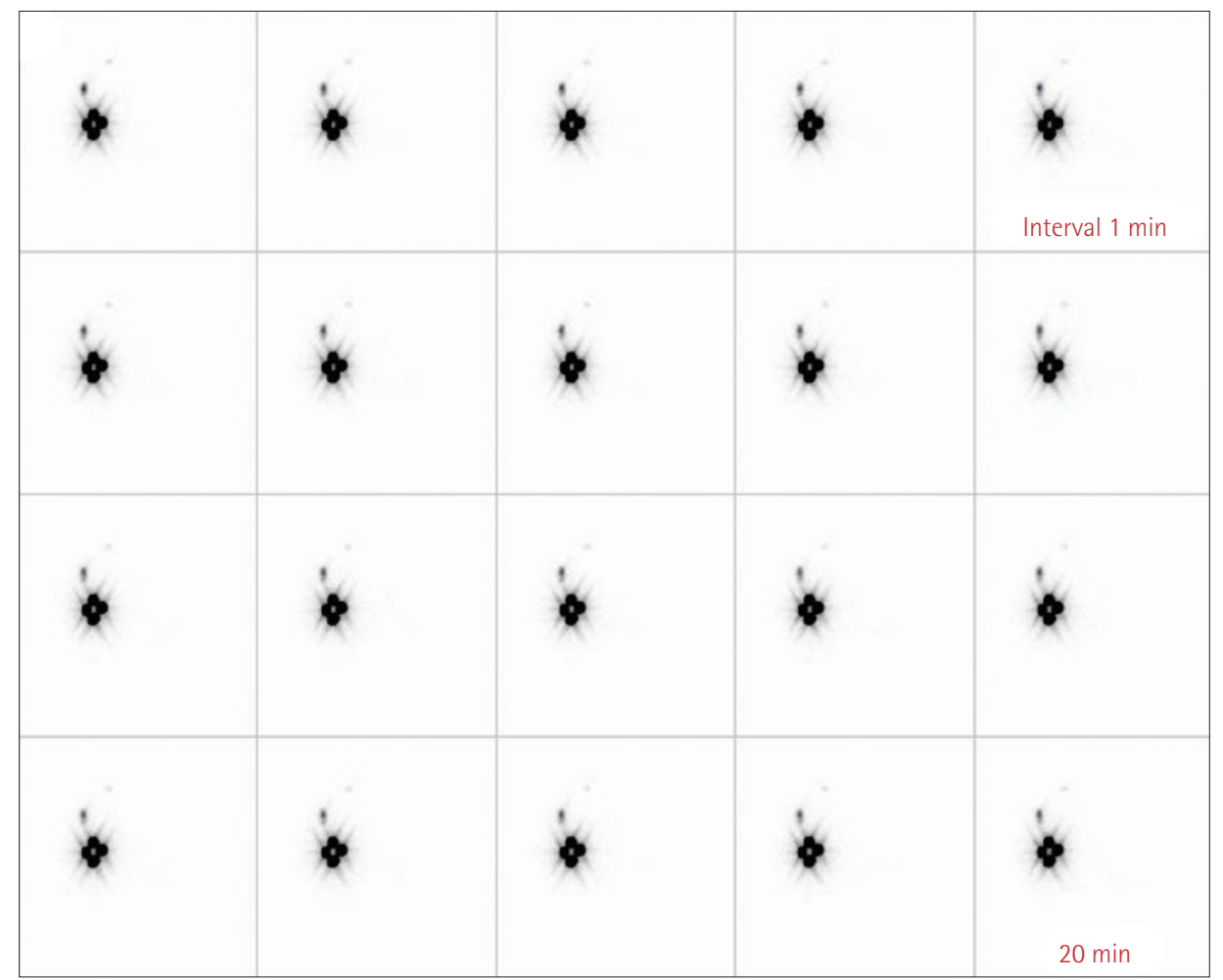

Fig. 2. Lymphoscintigraphy findings after injection of radioactive isotope (RI). After Rl injection, the drainage of sentinel lymph nodes were identified with lymphoscintigraphy every minute for 20 minutes.

tion rate, false-negative rate, concordance rate, and positive rate, were also collected. The SLN identification rate was defined as the detection rate of SLNs using a gamma probe intraoperatively in all patients injected with the RI. Therefore, we included two patients who underwent ALND without SLNB because of failure of SLN detection to calculate the identification rate to obtain an exact value. The false-negative case was defined as patients with pathologically negative SLNs and at least one pathologically positive nonSLN. In this study, we did not perform ALND in most cases involving negative SLNs on frozen biopsy. Therefore, we defined expected false-negative rate as the rate of patients with false-negative result plus patients with negative SLNs who did not undergo ALND and showed local axillary recurrence in the follow-up period. The concordance rate was defined as the rate of matching between frozen biopsy results and final pathology results. The positive rate was defined as the rate of patients with pathologically positive SLNs.

\section{SUVmax of the primary tumors on PET-CT}

The SUVmax of the primary tumors on PET-CT were recorded. A total of 14 patients were excluded from this analysis. Eight patients were not evaluated using PET-CT preoperatively. In addition, six patients who underwent PET-CT after a primary operation for ex- cision of a benign breast mass were diagnosed with malignancy on final pathology, and therefore, the SUVmax in these patients was 0 or minimal. Primary tumors in another six patients showed a similar degree of fluorodeoxyglucose (FDG) uptake as the blood pool, and the values were too minimal to calculate the SUV; thus, the SUVmax values of these patients were calculated as 0 .

\section{Statistical analysis}

Demographic and clinicopathologic data are presented as the frequency and percentage, and average values are presented as the mean \pm standard deviation. The values for evaluating the efficacy of SLNB, including the identification rate, expected false-negative rate, concordance rate, and positive rate, are presented as percentages and were compared with corresponding data from previous meta-analyses $[9,10,12]$. The Student t-test was used to compare SUVmax values between the SLN-negative and SLN-positive groups. The correlation between negative SLNs and SUVmax was analyzed using Pearson correlation coefficients. In addition, receiver operating characteristic (ROC) curves were used for determining the SUVmax cutoff for unnecessary ALN assessment. A P-value $<0.05$ was considered statistically significant. Statistical analyses were performed using IBM SPSS Statistics version 25.0 (IBM Corp., Armonk, NY, USA). 


\section{RESULTS}

Between March 2011 and May 2018, 142 patients who underwent SLNB were finally included in the analysis. The demographic and clinicopathologic characteristics of these patients are shown in Table 1. All patients enrolled in this study were women with a mean age of $55.9 \pm 10.8$ years (range, $24-86$ years). The histopathological

Table 1. Demographic and clinicopathologic characteristics of the patients

\begin{tabular}{|c|c|}
\hline Characteristics & Value $(n=142)$ \\
\hline Age (yr) & $55.9 \pm 10.8$ \\
\hline \multicolumn{2}{|l|}{ Menopausal status } \\
\hline Premenopausal & 48 (33.8) \\
\hline Postmenopausal & $94(66.2)$ \\
\hline \multicolumn{2}{|l|}{ Laterality } \\
\hline Right side & $58(40.8)$ \\
\hline Left side & 84 (59.2) \\
\hline Tumor size (cm) & $1.8 \pm 1.1$ \\
\hline \multicolumn{2}{|l|}{ T stage } \\
\hline pT1mi & $3(2.1)$ \\
\hline pT1a & $5(3.5)$ \\
\hline pT1b & $25(17.6)$ \\
\hline pT1c & $66(46.5)$ \\
\hline pT2 & $43(30.3)$ \\
\hline \multicolumn{2}{|l|}{ Histologic type } \\
\hline Invasive ductal carcinoma & $89(62.7)$ \\
\hline Invasive lobular carcinoma & $7(4.9)$ \\
\hline Invasive carcinoma with no special type & $34(23.9)$ \\
\hline Mucinous carcinoma & $6(4.2)$ \\
\hline Others & $6(4.2)$ \\
\hline \multicolumn{2}{|l|}{ Histologic grade } \\
\hline । & $29(20.4)$ \\
\hline$\|$ & $45(31.7)$ \\
\hline III & $68(47.9)$ \\
\hline \multicolumn{2}{|l|}{ ER } \\
\hline Positive & $113(79.6)$ \\
\hline Negative & $29(20.4)$ \\
\hline \multicolumn{2}{|l|}{$P R$} \\
\hline Positive & $101(71.1)$ \\
\hline Negative & $41(28.9)$ \\
\hline \multicolumn{2}{|l|}{ HER2 } \\
\hline 1 Positive & 27 (19.0) \\
\hline 2 Positive & 38 (26.8) \\
\hline 3 Positive & 33 (23.2) \\
\hline Negative & 44 (31.0) \\
\hline
\end{tabular}

Values are presented as mean \pm standard deviation or number (\%). ER, estrogen receptor; PR, progesterone receptor; HER2, human epidermal growth factor receptor 2 . diagnoses were as follows: invasive ductal carcinoma, 89 (62.7\%); invasive lobular carcinoma, seven (4.9\%); invasive carcinoma with no special type, 34 (23.9\%); mucinous carcinoma, six (4.2\%); and others, six (4.2\%). ER positivity and PR positivity were seen in 113 (79.6\%) and 101 patients (71.1\%), respectively, and HER2 positivity was seen in 33 patients (23.2\%) ( $3+$ considered as positive).

The average time from injection of an RI to operation was 198.7 minutes (range, 51-421 minutes). SLNs were successfully identified in 142 of 144 patients (98.6\%), and the mean number of harvested SLNs per patient was 3.5 (range, 1-9). ALND was performed in 38 of 142 patients (26.8\%), and 17 patients (12.0\%) had metastatic SLNs on frozen biopsy; 21 patients (14.8\%) underwent additional ALND, although SLN results were negative. The mean number of harvested ALNs, including SLNs, per patient was 14.6 (range, 6-36). Among 142 patients, 140 (98.6\%) had the same results on frozen biopsy and final pathology; two patients with positive SLNs on frozen biopsy showed negative SLNs on final pathology via hematoxylin and eosin and/or immunohistochemical staining. The median follow-up period in this study was 42.1 months, and there were no patients with negative SLNs and local axillary recurrence in the follow-up periods (Table 2).

\section{Association of the SUVmax of the primary tumor with SLN status}

Among 128 patients who underwent preoperative PET-CT, 111 were found to have negative SLNs and 17 were found to have positive SLNs. The SLN-negative group had a significantly lower mean SUVmax of the primary tumor than the SLN-positive group (2.60 \pm 2.32 vs. $4.38 \pm 2.60, \mathrm{P}=0.015$ ) (Table 3 ). The correlation between the SUVmax of the primary tumor and SLN status was statistically

Table 2. Results regarding the efficacy of sentinel lymph node biopsy

\begin{tabular}{lc}
\hline Efficacy & No. $(\%)$ \\
\hline Identification rate $(n=144)$ & $142(98.6)$ \\
Expected false-negative rate $(n=142)$ & 0 \\
Concordance rate $(n=142)$ & $140(98.6)$ \\
Positive rate $(n=142)$ & $17(12.0)$ \\
\hline
\end{tabular}

Table 3. Comparison of the SUVmax of the primary tumor according to SLN status

\begin{tabular}{lccc}
\hline SLN & No. & $\begin{array}{c}\text { SUVmax-T } \\
(\text { mean } \pm \text { SD })\end{array}$ & P-value \\
\hline SLN-negative & 111 & $2.60 \pm 2.32$ & 0.015 \\
SLN-positive & 17 & $4.38 \pm 2.60$ & \\
\hline
\end{tabular}

SUVmax, maximum standardized uptake value; T, primary tumor; SLN, sentinel lymph node; SD, standard deviation. 
significant $(\mathrm{r}=0.249, \mathrm{P}=0.005)$. ROC curve analysis was performed to determine the SUVmax cutoff for the primary tumor for diagnosing SLN metastasis (Fig. 3). The analysis revealed the area under the curve to be 0.707 (95\% confidence interval, 0.579-0.835; $\mathrm{P}=0.006$ ). The optimal cutoff value for negative SLN metastasis was 2.15, and its diagnostic performance was as follows: sensitivity, 82.4\%; specificity, $55.9 \%$; positive predictive value, $22.2 \%$; negative predictive value, 95.4\%; accuracy, 59.4\% (Table 4).

The patients enrolled in this analysis were classified according to molecular subtypes of breast cancer. Total 32 patients were luminal A type (ER and/or PR-positive, HER2-negative, Ki-67 < 14\%) and 68 patients were luminal B type (ER and/or PR-positive, HER2negative, Ki-67 $\geq 14 \%$ / ER and/or PR-positive, HER2-positive). There was no patient who showed SUVmax $<2.15$ and SLN metastasis in 12 patients with HER2-enriched type (ER and PR-negative, HER2-positive). Sixteen patients were basal-like type (ER and PR-negative, HER2-negative) and all of them showed negative SLN. The diagnostic performance of SUVmax using a cutoff of 2.15 according to molecular subtype were shown in Table 5.

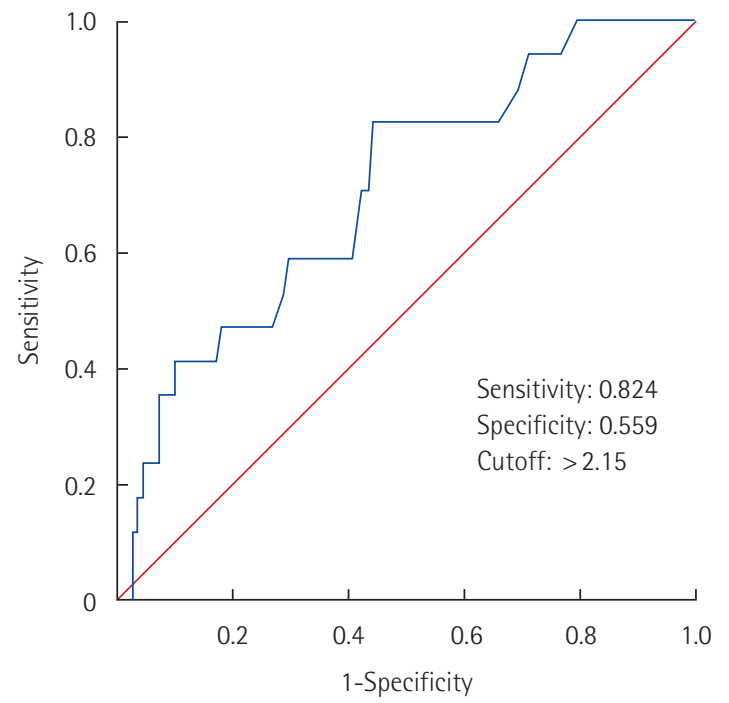

Fig. 3. Receiver operating characteristic curve for the maximum standardized uptake value of the primary tumor.

\section{DISCUSSION}

SLNB for breast cancer was first described in 1994 [22], and since then, several studies assessing the safety and efficacy of SLNB have been reported $[23,24]$. A few methods for SLNB have been described, including those using a vital dye, an RI, or both, and previous studies have reported that a combined method showed high efficiency and accuracy $[9,10,12]$. Miltenburg et al. [9] reviewed 912 cases and reported that using a radiocolloid and the combination of a radiocolloid and blue dye, the identification rates were $84.7 \%$ and $94.4 \%$, respectively, and the false-negative rates were $5.9 \%$ and $0 \%$, respectively. Use of the combined method resulted in a significantly higher identification rate than the use of blue dye or radiocolloid alone; however, there was no significant difference in the false-negative rate. Pesek et al. [10] and He et al. [12] also reported no significant difference in the false-negative rate between the combined method and the use of a radioisotope alone. In this study, the identification and false-negative rates with the use of an RI alone were not inferior to those obtained with the combined use of an RI and blue dye in previous meta-analyses $[9,10,12]$ (identification rate, $98.6 \%$ vs. $94.4 \%$; false-negative rate, $0 \%$ vs. $0 \%$ ). The method using an RI alone showed slightly lower concordance and positive rates than the combined method used in a previous study [9] (concordance rate, 98.6\% vs. 100\%; positive rate, $12.0 \%$ vs. $19.0 \%$ ) (Table 6), but these values were not directly associated with the efficacy of SLNB.

Some prospective studies conducted in Korea for evaluating the

Table 4. Diagnostic performance of the SUVmax of the primary tumor for detecting sentinel lymph node metastasis using a cutoff of 2.15

\begin{tabular}{lc}
\hline Test & Rate (\%) \\
\hline Sensitivity & 82.4 \\
Specificity & 55.9 \\
Positive predictive value & 22.2 \\
Negative predictive value & 95.4 \\
Accuracy & 59.4 \\
\hline
\end{tabular}

SUVmax, maximum standardized uptake value.

Table 5. Diagnostic performance of the SUVmax of the primary tumor using cutoff of 2.15 according to molecular subtypes of breast cancer

\begin{tabular}{lcccc}
\hline Test & Luminal A (\%) & Luminal B (\%) & HER2-enriched (\%) & Basal-like (\%) \\
\hline Sensitivity & 22.2 & 34.4 & 11.1 & 0 \\
Specificity & 95.7 & 94.4 & 100 & 100 \\
Positive predictive value & 66.7 & 84.6 & 100 & 0 \\
Negative predictive value & 75.9 & 61.8 & 27.3 & 18.8 \\
Accuracy & 75 & 66.2 & 33.3 & 18.8 \\
\hline
\end{tabular}

SUVmax, maximum standardized uptake value; HER2, human epidermal growth factor receptor 2. 
Table 6. Comparison of the efficacy of sentinel lymph node biopsy between our study and previous studies

\begin{tabular}{|c|c|c|c|c|c|c|c|}
\hline Author (year) & No. & No. of SLNs ${ }^{a)}$ & No. of $A L N s^{a)}$ & $\begin{array}{c}\text { Identification } \\
\text { rate }(\%)\end{array}$ & $\begin{array}{c}\text { False-negative } \\
\text { rate }(\%)\end{array}$ & $\begin{array}{c}\text { Concordance } \\
\text { rate }(\%)\end{array}$ & $\begin{array}{l}\text { Positive } \\
\text { rate (\%) }\end{array}$ \\
\hline Current study & 142 & $3.5(1-9)$ & $14.6(6-36)$ & 98.6 & 0 & 98.6 & 12 \\
\hline Miltenburg et al. (1999) [9] & 912 & $1.9(1.3-2.2)$ & $19.5(9.4-28.1)$ & 94.4 & 0 & 100 & 19 \\
\hline Pesek et al. (2012) [10] & 9,306 & & & & 5.9 & & \\
\hline He et al. (2016) [12] & 15,462 & & & & 7.5 & & \\
\hline
\end{tabular}

SLN, sentinel lymph node; ALN, axillary lymph node.

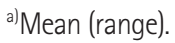

efficacy of SLNB with an RI alone have shown high identification rates of $94.4 \%-99.1 \%$ and varying false-negative rates of $4.1 \%-$ $16.7 \%$ [25-27]. In comparison with those of previous studies, the results of this study were not inferior, and thus, SLNB with an RI alone may be considered a reasonable option.

Unlike other studies, the present study used long-term outcomes to evaluate the oncologic safety of SLNB with an RI alone. There were some patients with local axillary recurrence, but all of them had been diagnosed with metastatic ALNs at the time of the operation. Furthermore, there was no false-negative case involving the detection of local axillary recurrence during at least 24 months of follow-up. These results indicate the long-term safety of the single agent method and suggest that it is a feasible option for SLNB.

Another objective of this study was to determine the correlation between the SUVmax of the primary tumor and SLN metastasis. Ueda et al. [28] and Song et al. [29] reported that high SUVmax of the primary tumor on preoperative PET-CT was related to ALN metastasis. Refaat et al. [19] also reported the correlation between FDG uptake and ALN metastasis in a prospective study and suggested the SUVmax cutoff for the primary tumor for detecting ALN metastasis to be 4.2, which was higher than our cutoff of 2.15; this may be due to differences in patient selection. We analyzed patients with clinically negative ALNs, and our study had a relatively small sample size compared with the previous study including 198 patients.

This study had several limitations. It was a retrospective study; therefore, the data were fully dependent on medical records. Therefore, there may have been inaccuracies in the causes for ALND without SLNB. Second, there was no control group that underwent SLNB using other methods, such as SLNB with blue dye or a combination of blue dye and an RI; thus, we compared our results with those of previous studies. Third, we used an RI alone for SLNB, and we excised lymph nodes with at least some radioactivity; therefore, SLNs were harvested extensively, and the number of harvested lymph nodes was relatively high. It is considered that if the time interval is short, the RI is not sufficiently drained into the SLNs; thus, SLNs were harvested extensively for proper SLNB. Additionally, the expected false-negative rate of SLNB was calculated indirectly. ALND is essential to confirm false-negative cases; however, many patients did not undergo ALND because of negative SLNs in this study. Therefore, we estimated the expected false-negative rate from the results of local axillary recurrence in patients who did not undergo ALND. Finally, it is difficult to generalize the results of the correlation between primary tumor SUVmax and SLN metastasis. Because the methods of measuring SUVmax could differ according to hospital protocols, and the SUVmax could be influenced by many biological and technological factors including weight composition, blood glucose level, uptake time of the tracer, and reconstruction parameters [30]. And the current Korean National Health Insurance system does not recommend preoperative PET-CT scan for early node-negative breast cancer patients, so routine evaluation of SUVmax could be difficult in all patients. Moreover, only patients with clinically negative ALNs were included in this study with the positive rate of $12.0 \%$. Therefore, the data of patients with ALN metastasis should be included for exact results, and further large-scale studies including patients regardless of their ALN status are required.

In conclusion, SLNB with an RI alone can yield comparable identification rates in breast cancer patients compared with SLNB using the combined method shown in previous studies, and it is oncologically safe with respect to long-term outcomes. The single agent method using an RI is not inferior to other methods and serves as a feasible option for SLNB. Furthermore, the SUVmax of the primary tumor on PET-CT was correlated with SLN status, especially when the SUVmax was $<2.15$. Thus, the number of excised SLNs could be minimized for preventing complications of SLNB and improving quality of life of patients when the SUVmax of the primary tumor is extremely low. Further large prospective cohort studies are needed to confirm the reliability of SUVmax for the prediction of ALN status.

\section{CONFLICT OF INTEREST}

No potential conflict of interest relevant to this article was reported. 


\section{REFERENCES}

1. DeSantis CE, Bray F, Ferlay J, Lortet-Tieulent J, Anderson BO, Jemal A. International variation in female breast cancer incidence and mortality rates. Cancer Epidemiol Biomarkers Prev 2015;24: 1495-506.

2. Bray F, McCarron P, Parkin DM. The changing global patterns of female breast cancer incidence and mortality. Breast Cancer Res 2004;6:229-39.

3. Chatterjee A, Serniak N, Czerniecki BJ. Sentinel lymph node biopsy in breast cancer: a work in progress. Cancer J 2015;21:7-10.

4. Veronesi U, Paganelli G, Viale G, Luini A, Zurrida S, Galimberti V, et al. A randomized comparison of sentinel-node biopsy with routine axillary dissection in breast cancer. N Engl J Med 2003;349: 546-53.

5. Schijven MP, Vingerhoets AJ, Rutten HJ, Nieuwenhuijzen GA, Roumen RM, van Bussel ME, et al. Comparison of morbidity between axillary lymph node dissection and sentinel node biopsy. Eur J Surg Oncol 2003;29:341-50.

6. Lucci A, McCall LM, Beitsch PD, Whitworth PW, Reintgen DS, Blumencranz PW, et al. Surgical complications associated with sentinel lymph node dissection (SLND) plus axillary lymph node dissection compared with SLND alone in the American College of Surgeons Oncology Group Trial Z0011. J Clin Oncol 2007;25: 3657-63.

7. Hsueh EC, Giuliano AE. Sentinel lymph node technique for staging of breast cancer. Oncologist 1998;3:165-70.

8. Ferreira P, Baia R, Antonio A, Almeida J, Simoes J, Amaro J, et al. Sentinel lymph node biopsy: technique validation at the Setubal Medical Centre, Portugal. Ecancermedicalscience 2009;3:124.

9. Miltenburg DM, Miller C, Karamlou TB, Brunicardi FC. Meta-analysis of sentinel lymph node biopsy in breast cancer. J Surg Res 1999;84:138-42.

10. Pesek S, Ashikaga T, Krag LE, Krag D. The false-negative rate of sentinel node biopsy in patients with breast cancer: a meta-analysis. World J Surg 2012;36:2239-51.

11. Li J, Chen X, Qi M, Li Y. Sentinel lymph node biopsy mapped with methylene blue dye alone in patients with breast cancer: a systematic review and meta-analysis. PLoS One 2018;13:e0204364.

12. He PS, Li F, Li GH, Guo C, Chen TJ. The combination of blue dye and radioisotope versus radioisotope alone during sentinel lymph node biopsy for breast cancer: a systematic review. BMC Cancer 2016;16:107.

13. Wong SL, Edwards MJ, Chao C, Tuttle TM, Noyes RD, Carlson DJ, et al. Sentinel lymph node biopsy for breast cancer: impact of the number of sentinel nodes removed on the false-negative rate. J Am Coll Surg 2001;192:684-91.
14. Raut CP, Hunt KK, Akins JS, Daley MD, Ross MI, Singletary SE, et al. Incidence of anaphylactoid reactions to isosulfan blue dye during breast carcinoma lymphatic mapping in patients treated with preoperative prophylaxis: results of a surgical prospective clinical practice protocol. Cancer 2005; 104:692-9.

15. Mathelin C, Croce S, Brasse D, Gairard B, Gharbi M, Andriamisandratsoa N, et al. Methylene blue dye, an accurate dye for sentinel lymph node identification in early breast cancer. Anticancer Res 2009;29:4119-25.

16. Singh-Ranger G, Mokbel K. Capsular contraction following immediate reconstructive surgery for breast cancer: an association with methylene blue dye. Int Semin Surg Oncol 2004;1:3.

17. Futamura M, Asano T, Kobayashi K, Morimitsu K, Nawa M, Kanematsu M, et al. Prediction of macrometastasis in axillary lymph nodes of patients with invasive breast cancer and the utility of the SUV lymph node/tumor ratio using FDG-PET/CT. World J Surg Oncol 2015;13:49.

18. Park J, Byun BH, Noh WC, Lee SS, Kim HA, Kim EK, et al. Lymph node to primary tumor SUV ratio by 18 F-FDG PET/CT and the prediction of axillary lymph node metastases in breast cancer. Clin Nucl Med 2014;39:e249-53.

19. Refaat R, Abd Alkhalik Basha M, Sobhi Hassan M, Hussein RS, Al-Molla RM, Awad NM, et al. Is FDG maximum standardized uptake value of primary breast cancer a prognostic factor for locoregional axillary lymph node metastasis? Acta Radiol 2019;60: 1241-50.

20. Somner JE, Dixon JM, Thomas JS. Node retrieval in axillary lymph node dissections: recommendations for minimum numbers to be confident about node negative status. J Clin Pathol 2004;57:845-8.

21. Vujovic O, Yu E, Cherian A, Dar AR, Stitt L, Perera F. The number of axillary nodes removed as a predictor of regional recurrence in node negative breast cancer. Radiother Oncol 2009;91:38-41.

22. Giuliano AE, Kirgan DM, Guenther JM, Morton DL. Lymphatic mapping and sentinel lymphadenectomy for breast cancer. Ann Surg 1994;220:391-401.

23. Canavese G, Bruzzi P, Catturich A, Tomei D, Carli F, Garrone E, et al. Sentinel lymph node biopsy versus axillary dissection in node-negative early-stage breast cancer: 15-year follow-up update of a randomized clinical trial. Ann Surg Oncol 2016;23:2494-500.

24. Canavese G, Catturich A, Vecchio C, Tomei D, Gipponi M, Villa G, et al. Sentinel node biopsy compared with complete axillary dissection for staging early breast cancer with clinically negative lymph nodes: results of randomized trial. Ann Oncol 2009;20:1001-7.

25. Kim HA, Lee JH, Moon BI, Choi KJ, Sung SH, Choi HY, et al. The efficacy of sentinel node biopsy using subareolar injection of radioactive material in breast cancer. J Korean Breast Cancer Soc 2003; 6:180-5. 
26. Kim HJ, Chang MA, Hong SJ, Lee JS, Jung MS, Kim MJ, et al. Result of sentinel lymph node biopsy using radioisotope in clinically lymph node negative breast cancer. J Breast Cancer 2007;10:141-6.

27. Lee HD, Kim DY, Choi JW, Park BW, Jung WH, Kim HJ, et al. Sentinel lymph node biopsy using radioactive material in breast cancer patients. J Korean Breast Cancer Soc 2000;3:104-14.

28. Ueda S, Tsuda H, Asakawa H, Shigekawa T, Fukatsu K, Kondo N, et al. Clinicopathological and prognostic relevance of uptake level using 18F-fluorodeoxyglucose positron emission tomography/ computed tomography fusion imaging (18F-FDG PET/CT) in primary breast cancer. Jpn J Clin Oncol 2008;38:250-8.

29. Song BI, Hong CM, Lee HJ, Kang S, Jeong SY, Kim HW, et al. Prognostic value of primary tumor uptake on F-18 FDG PET/CT in patients with invasive ductal breast cancer. Nucl Med Mol Imaging 2011;45:117-24.

30. Adams MC, Turkington TG, Wilson JM, Wong TZ. A systematic review of the factors affecting accuracy of SUV measurements. AJR Am J Roentgenol 2010;195:310-20. 